This is the author's copy of the publication as archived with the DLR's electronic library at http://elib.dlr.de. Please consult the original publication for citation.

\title{
Joint-Level Control of the DLR Lightweight Robot SARA
}

Maged Iskandar, Christian Ott, Oliver Eiberger, Manuel Keppler, Alin Albu-Schäffer, and Alexander Dietrich

\section{Copyright Notice}

(C)2020 IEEE. Personal use of this material is permitted. However, permission to reprint/republish this material for advertising or promotional purposes or for creating new collective works for resale or redistribution to servers or lists, or to reuse any copyrighted component of this work in other works must be obtained from the IEEE.

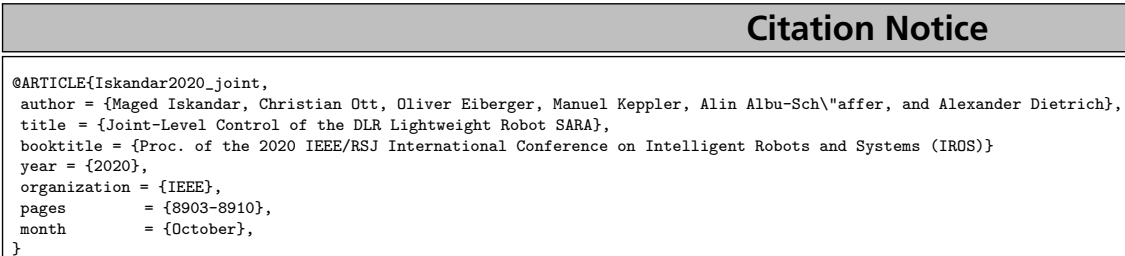




\title{
Joint-Level Control of the DLR Lightweight Robot SARA
}

\author{
Maged Iskandar, Christian Ott, Oliver Eiberger, Manuel Keppler, Alin Albu-Schäffer, and Alexander Dietrich
}

\begin{abstract}
Lightweight robots are known to be intrinsically elastic in their joints. The established classical approaches to control such systems are mostly based on motor-side coordinates since the joints are comparatively stiff. However, that inevitably introduces errors in the coordinates that actually matter: the ones on the link side. Here we present a new joint-torque controller that uses feedback of the link-side positions. Passivity during interaction with the environment is formally shown as well as asymptotic stability of the desired equilibrium in the regulation case. The performance of the control approach is experimentally validated on DLR's new generation of lightweight robots, namely the SARA robot, which enables this step from motor-side-based to link-sided-based control due to sensors with higher resolution and improved sampling rate.
\end{abstract}

\section{INTRODUCTION}

Nowadays, the growing interest in lightweight collaborative robots becomes more evident in many applications. A challenge that is inherent to these modern systems is the joint flexibility. The main source of flexibility is due to the presence of a flexible reduction element (e.g. Harmonic drive gears or other transmission units). These transmission elements have the advantage of high reduction ratios while enabling compact in-line design. However, the weakly damped elasticity results in an unwanted oscillatory behavior of the output (link position). In order to achieve a high performance on these systems, it is crucial to include this parasitic elasticity in the control design. If not handled properly, one faces vibration problems during free motion and possibly chattering/instability in scenarios when the robot interacts with the environment [1]. In order to enable safe interaction with the environment and in particular with humans, these lightweight robots are often equipped with torque sensors in addition to motor position sensors [2], [3]. Compared to pure motor position sensing, the additional integration of torque sensors provides various advantages from the control point of view. The combined feedback of the motor position and joint torque measurements in a feedback loop allows to improve active vibration damping and to achieve a desired impedance behavior during physical human-robot interaction [4], [2]. In [5] this combined feedback is used to solve the position, torque, and impedance control problem. It is important to remark that the additional integration of torque sensors introduces another source of elasticity. However, this effect can be lumped together with the flexibility introduced by the transmission gear into concentrated flexibility for each joint.

The link-side regulation problem for flexible joint robots has been treated in [6]-[9]. All of these controllers consider

All authors are with the DLR - German Aerospace Center, Robotics and Mechatronics Center, Institute of Robotics and Mechatronics, Wessling, Germany. Email: maged.iskandar@dlr.de

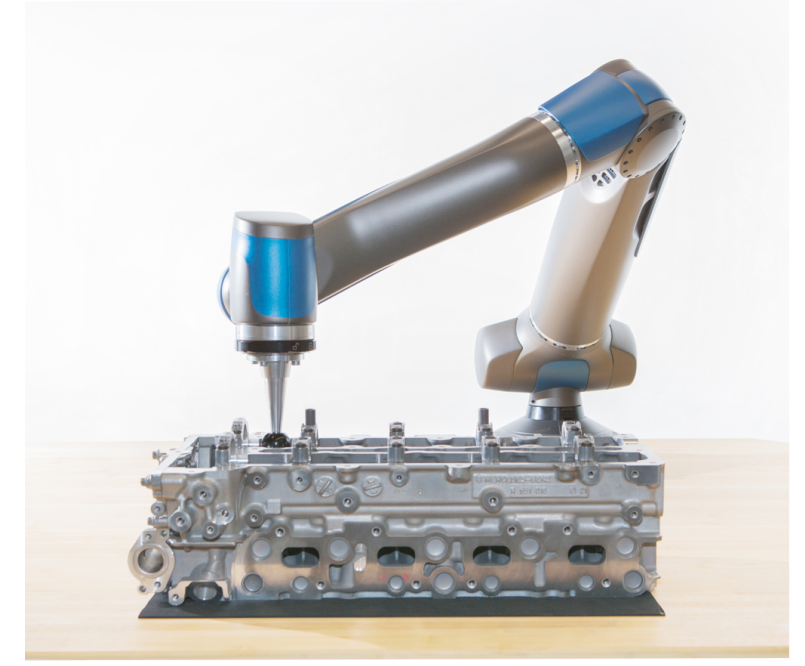

Fig. 1: The next generation of DLR lightweight robots: SARA (Safe Autonomous Robotic Assistant)

only feedback of control-input-collocated variables. Therefore, the damping performance is lower compared to the regulation controllers reported in [10], [11], which employ feedback of control-input-noncollocated variables. However, no stability analysis of the closed-loop dynamics has been provided so far.

The proposed approach follows the idea of a change of coordinates as introduced in [12]. This control concept preserves the intrinsic structure of robots with elastic joints while achieving a desired link-side motion tracking behavior. An extension [13] of this concept allows to implement a torque interface for the compliant robots which can be employed to implement a desired Cartesian impedance behavior at the TCP. In this work, we modify this concept to tailor it to the special characteristics of lightweight robots with additional joint torque sensors. The object of this work is to derive a torque-based controller that effectively dampens joint vibrations and provides a link-side torque interface while exploiting the full set of available measurements including joint torques.

We use the state vector $(\boldsymbol{q}, \boldsymbol{\tau}, \dot{\boldsymbol{q}}, \dot{\boldsymbol{\tau}})$ for control throughout the paper. It contains the link positions $q$ and the joint torques $\tau$, both directly measured by sensors, as well as their first derivatives $\dot{\boldsymbol{q}}$ and $\dot{\boldsymbol{\tau}}$, which are computed numerically. Let us motivate this choice of state vector. Obviously, in absence of a torque sensor, the state vector $(\boldsymbol{q}, \boldsymbol{\theta}, \dot{\boldsymbol{q}}, \dot{\boldsymbol{\theta}})$, as in the work [12], can be used instead. ${ }^{1}$ However, accurate values of the joint torques are crucial for good control performance. Model-based joint torque estimation is potentially insuffi-

\footnotetext{
${ }^{1}$ These two state vectors are related in a linear fashion.
} 
cient for robots with relatively high joint stiffness values as present in lightweight robots. Note that the resolution of the torque signal is directly related to uncertainties in the joint stiffness estimation or joint deflection measurement. In robots where elasticity is deliberately introduced into the joints in order to increase the interaction performance and safety, the joint stiffness is usually of magnitudes lower than in lightweight robots. In this case, it is sufficient to rely on feedback of the state $(\boldsymbol{q}, \boldsymbol{\theta}, \dot{\boldsymbol{q}}, \dot{\boldsymbol{\theta}})$ including joint torque signals.

The paper is organized as follows. In Section II we introduce the system model to be used throughout this work and provide an overview of the standard control approaches for this type of system. The proposed method and the general design idea are presented in Section III. In Section IV, a formal passivity and stability analysis is provided. Experimental validations of the approach are shown and discussed in Section V. Finally, the conclusion in Section VI closes the paper.

\section{BACKGROUND}

The equations of motion of a flexible-joint robot require twice the number of generalized coordinates compared to the rigid-body case. Correspondingly, different coordinates are associated with the motor and the link side, respectively, see Fig. 2. The dynamics of a robot with elastic joints based on the standard model [14] can be written as

$$
\begin{aligned}
\boldsymbol{M}(\boldsymbol{q}) \ddot{\boldsymbol{q}}+\boldsymbol{C}(\boldsymbol{q}, \dot{\boldsymbol{q}}) \dot{\boldsymbol{q}}+\boldsymbol{g}(\boldsymbol{q}) & =\boldsymbol{K}(\boldsymbol{\theta}-\boldsymbol{q})+\boldsymbol{\tau}_{\text {ext }}, \\
\boldsymbol{B} \ddot{\boldsymbol{\theta}}+\boldsymbol{K}(\boldsymbol{\theta}-\boldsymbol{q}) & =\boldsymbol{\tau}_{m} .
\end{aligned}
$$

Herein, $\boldsymbol{q} \in \mathbb{R}^{n}$ represents the vector of the $n$ link-side positions and $\boldsymbol{\theta} \in \mathbb{R}^{n}$ describes the motor positions. ${ }^{2}$ Let $\boldsymbol{\tau}=\boldsymbol{K}(\boldsymbol{\theta}-\boldsymbol{q})$ be the elastic torque which is transmitted between motor and link, with the positive definite joint stiffness $\boldsymbol{K} \succ 0$. Gravitational effects are represented by $\boldsymbol{g}(\boldsymbol{q}) \in \mathbb{R}^{n}$, and the link-side inertia and Coriolis/centrifugal matrices are defined by $\boldsymbol{M}(\boldsymbol{q}) \in \mathbb{R}^{n \times n}$ and $\boldsymbol{C}(\boldsymbol{q}, \dot{\boldsymbol{q}}) \in \mathbb{R}^{n \times n}$, respectively. The external torque is described by $\boldsymbol{\tau}_{\text {ext }}$. The motor inertia is given by $\boldsymbol{B} \in \mathbb{R}^{n \times n}$ and accelerated by the motor torque $\tau_{m} .{ }^{3}$ The above-mentioned model is considered to be simplified as it was proposed in [14] in the sense that it assumes that the kinetic energy of each motor is only due to its own rotation. Two additional common assumptions have been made with respect to (1)-(2):

Assumption 1: The joint elasticity can be modeled as a linear torsional spring.

Assumption 2: The flexibility is concentrated at the robot joints while the links are rigid.

Probably the most common way for torque-regulation control (1)-(2) is to utilize a classical PD controller [1], [5]

$$
\boldsymbol{\tau}_{m}=\boldsymbol{\tau}_{d}+\boldsymbol{K}_{T}\left(\boldsymbol{\tau}_{d}-\boldsymbol{\tau}\right)-\boldsymbol{K}_{S} \dot{\boldsymbol{\tau}}
$$

\footnotetext{
${ }^{2}$ The motor position is reflected with the reduction gear ratio so that it has the same dynamic range as the link position, and the model (1)-(2) is consequently independent of the gear ratio.

${ }^{3}$ The motor is modeled as an ideal torque source neglecting the electrical dynamics.
}

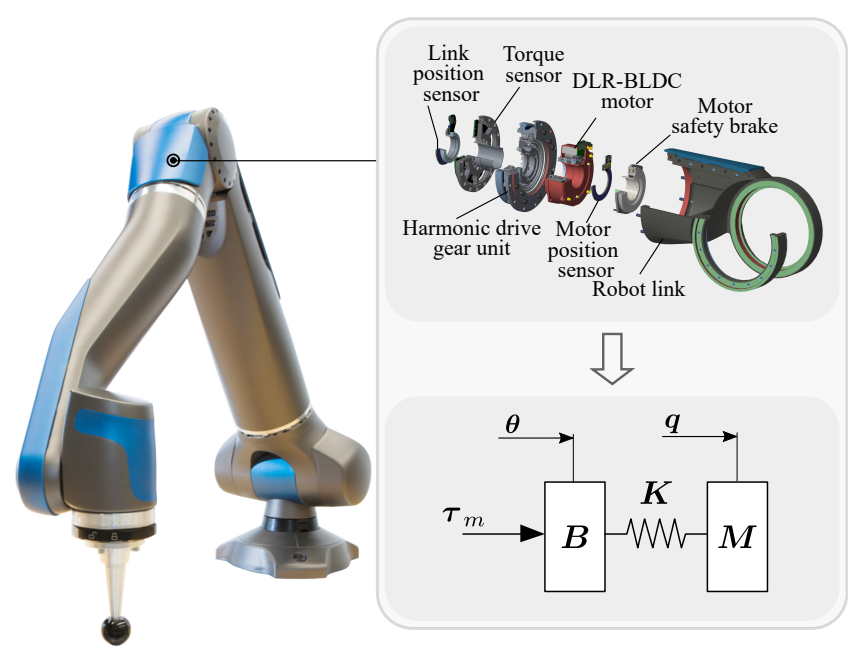

Fig. 2: The DLR SARA robot, an exploded view of a single joint design, and the simplified flexible joint model.

The desired joint torque $\tau_{d}$ is fed forward and the (measured) joint torque $\tau$ and its time derivative $\dot{\tau}$ are used in the feedback loop incorporating the corresponding gains $\boldsymbol{K}_{T}$, $\boldsymbol{K}_{S} \cdot{ }^{4}$ From a physical point of view, (3) can be intuitively interpreted as an active reduction of the motor inertia from $\boldsymbol{B}$ to the desired value $\boldsymbol{B}_{d}$ when choosing $\boldsymbol{K}_{T}=\boldsymbol{B} \boldsymbol{B}_{d}^{-1}-\boldsymbol{I}$, as shown in [5], [9]. The control action (3) can be combined with a motor position PD regulation controller to form the so-called full-state feedback controller

$$
\boldsymbol{\tau}_{d}=-\boldsymbol{K}_{\theta} \tilde{\boldsymbol{\theta}}-\boldsymbol{D}_{\theta} \dot{\boldsymbol{\theta}}+\boldsymbol{g}(\boldsymbol{\theta})
$$

where $\tilde{\boldsymbol{\theta}}=\boldsymbol{\theta}-\boldsymbol{\theta}_{d}$ is the motor position error, and $\boldsymbol{\theta}_{d}$ is the desired, constant motor position. Furthermore, $\boldsymbol{K}_{\theta}, \boldsymbol{D}_{\theta} \succ 0$ represent the controller stiffness and damping on the motor side. To implement a desired link-side behavior with (4), $\boldsymbol{\theta}_{d}$ can be statically computed model-based on the desired linkside position $\boldsymbol{q}_{d}$ utilizing information on the joint elasticity and gravitational effects. However, the performance of this controller is highly dependent on uncertainties in these quantities. In the model (1)-(2), dissipative friction effects can be included in different ways. The most dominant component in SARA is the motor-side friction due to the harmonic drive gear. However, motor-side friction is considerably reduced as a result of the motor inertia shaping as described later in Sect. III-C. Further friction effects can be addressed by means of model-based friction compensation techniques [15]-[17] and/or the use of motor disturbance observers [18]. Furthermore, structural damping can be considered as the result of a viscoelastic joint model which can be, in turn, reflected as a compensation term in the control law [5]. Nevertheless, the initial design concept in this paper focuses on the effects of joint flexibility, and therefore, friction effects are not explicitly considered in the following analysis.

\section{METHODS}

The aim of this work is to achieve the same performance in terms of end-effector motion accuracy as in pure rigid-

\footnotetext{
${ }^{4}$ The term $\dot{\tau}$ is usually derived numerically, based on $\tau$.
} 
body robots where the joint actuator is acting (applying the actuation torque) directly on the link coordinates $\boldsymbol{q}$. The main challenge is described by the fact that the motor torque (control input) is non-collocated with the link coordinates (output).

\section{A. Link-side torque interface and motor inertia shaping}

Since the goal is to explicitly control the link side, the desired joint torque $\tau_{d}$ is introduced on the link side. Moreover, the motor inertia shall be actively reduced from $\boldsymbol{B}$ to $\boldsymbol{B}_{d}$. Figure 3 illustrates these modifications. Inspired by [12], we introduce a new set of motor coordinates $\boldsymbol{\eta}$. We aim for a closed-loop dynamics of the form

$$
\begin{aligned}
\boldsymbol{M}(\boldsymbol{q}) \ddot{\boldsymbol{q}}+\boldsymbol{C}(\boldsymbol{q}, \dot{\boldsymbol{q}}) \dot{\boldsymbol{q}}+\boldsymbol{g}(\boldsymbol{q}) & =\boldsymbol{K}(\boldsymbol{\eta}-\boldsymbol{q})+\boldsymbol{\tau}_{d} \\
\boldsymbol{B}_{d} \ddot{\boldsymbol{\eta}}+\boldsymbol{K}(\boldsymbol{\eta}-\boldsymbol{q}) & =\mathbf{0}
\end{aligned}
$$

with the corresponding relation

$$
\boldsymbol{K}(\boldsymbol{\theta}-\boldsymbol{q})=\boldsymbol{K}(\boldsymbol{\eta}-\boldsymbol{q})+\boldsymbol{\tau}_{d}
$$

required to transform the original dynamics (1)-(2) to the desired dynamics (5)-(6). Let $\boldsymbol{\eta}$ therefore be called the virtual motor coordinate. To obtain the control action for $\tau_{m}$, (7) is differentiated twice, and $\ddot{\theta}$ as well as $\ddot{\boldsymbol{\eta}}$ are inserted from (2) and (6), respectively. That yields

$$
\boldsymbol{\tau}_{m}=\boldsymbol{\tau}_{d}+\left(\boldsymbol{B} \boldsymbol{B}_{d}^{-1}-\boldsymbol{I}\right)\left(\boldsymbol{\tau}_{d}-\boldsymbol{\tau}\right)+\boldsymbol{B} \boldsymbol{K}^{-1} \ddot{\boldsymbol{\tau}}_{d} .
$$

Interestingly, (8) can be regarded as a proportional torque controller with feedforward action of $\tau_{d}$, but with an additional feedforward component that also contains the second derivative of $\tau_{d}$. The torque feedback can be physically interpreted as the implication of inertia scaling/shaping or modification of the effective motor inertia, respectively. If $\boldsymbol{B}>\boldsymbol{B}_{d}$ holds true, this indicates that the apparent inertia

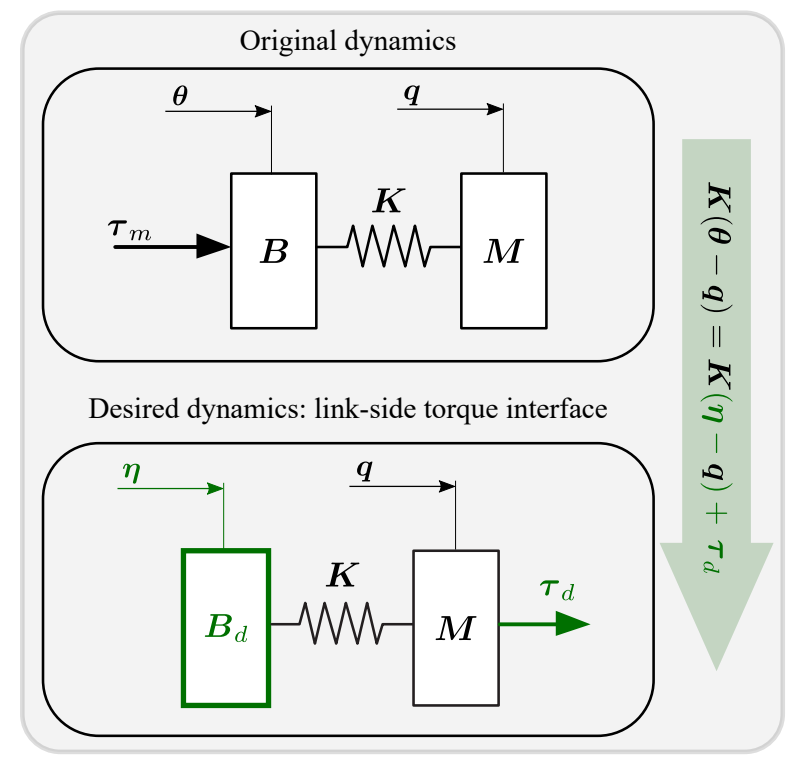

Fig. 3: The design concept of the joint-level torque control depicted for a simplified single-joint model. The original dynamics (top) and desired dynamics (bottom) are shown.

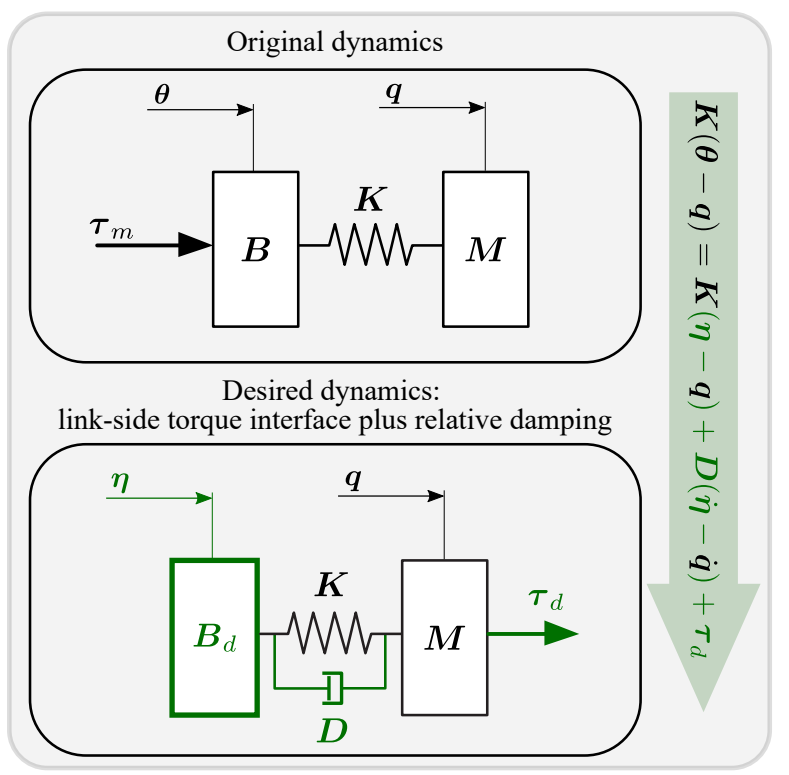

Fig. 4: The original dynamics and desired dynamics in case of shaping the inertia to $\boldsymbol{B}_{d}$ and providing link-side torque interface $\tau_{d}$ as well as adding relative damping $\boldsymbol{D}$ between the scaled motor inertia and the link inertia.

of the motor is scaled down, which means that the closedloop system will react to external forces as if the smaller inertia $\boldsymbol{B}_{d}$ is physically present.

This intermediate step presented in Section III-A provides the new control input $\tau_{d}$, which can be used to apply any control strategy on the link side.

B. Link-side torque interface, motor inertia shaping, and relative damping

However, since vibration suppression between motor and the link motion is of major relevance for the overall control performance, it is beneficial to dissipate energy at this very location, that is, by an additional relative damper as shown in Fig. 4 (bottom).

Analogous to Section III-A, one can formulate the corresponding equations of motion 5

$$
\begin{aligned}
& \boldsymbol{M} \ddot{\boldsymbol{q}}+\boldsymbol{C} \dot{\boldsymbol{q}}+\boldsymbol{g}=\boldsymbol{K}(\boldsymbol{\eta}-\boldsymbol{q})+\boldsymbol{D}(\dot{\boldsymbol{\eta}}-\dot{\boldsymbol{q}})+\boldsymbol{\tau}_{d}, \\
& \boldsymbol{B}_{d} \ddot{\boldsymbol{\eta}}+\boldsymbol{K}(\boldsymbol{\eta}-\boldsymbol{q})+\boldsymbol{D}(\dot{\boldsymbol{\eta}}-\dot{\boldsymbol{q}})=\mathbf{0},
\end{aligned}
$$

and the necessary condition for the coordinate transformation

$$
\boldsymbol{K}(\boldsymbol{\theta}-\boldsymbol{q})=\boldsymbol{K}(\boldsymbol{\eta}-\boldsymbol{q})+\boldsymbol{D}(\dot{\boldsymbol{\eta}}-\dot{\boldsymbol{q}})+\boldsymbol{\tau}_{d}
$$

between $\boldsymbol{\theta}$ and $\boldsymbol{\eta}$, similar to (7). The additional damper is characterized by the damping matrix $\boldsymbol{D} \succ 0$. To obtain the control law required to achieve (9)-(10), the second derivative of (11) w.r.t. time can be utilized:

$$
\boldsymbol{D} \boldsymbol{\eta}^{(3)}+\boldsymbol{K} \ddot{\boldsymbol{\eta}}=\boldsymbol{K} \ddot{\boldsymbol{\theta}}+\boldsymbol{D} \boldsymbol{q}^{(3)}-\ddot{\boldsymbol{\tau}}_{d}
$$

Now it is observable that the original motor acceleration appears in (12). Thus, one can solve (12) for $\tau_{m}$ by substituting $\ddot{\boldsymbol{\theta}}$ from (2). In (12), the virtual motor acceleration $\ddot{\boldsymbol{\eta}}$ and the

\footnotetext{
${ }^{5}$ Note that dependencies on the states have been omitted for the sake of readability.
} 
jerk $\boldsymbol{\eta}^{(3) 6}$ can be straightforwardly obtained from (10) and its time derivative, respectively. That finally delivers

$$
\begin{aligned}
\boldsymbol{\tau}_{m}= & \boldsymbol{B} \boldsymbol{K}^{-1} \boldsymbol{D} \boldsymbol{B}_{d}^{-1}(-\boldsymbol{K}(\dot{\boldsymbol{\eta}}-\dot{\boldsymbol{q}})-\boldsymbol{D}(\ddot{\boldsymbol{\eta}}-\ddot{\boldsymbol{q}})) \\
& +\boldsymbol{B} \ddot{\boldsymbol{\eta}}+\boldsymbol{B} \boldsymbol{K}^{-1}\left(\ddot{\boldsymbol{\tau}}_{d}-\boldsymbol{D} \boldsymbol{q}^{(3)}\right)+\boldsymbol{K}(\boldsymbol{\theta}-\boldsymbol{q}) .
\end{aligned}
$$

Substituting $\ddot{\eta}$ from (10) and $\dot{\eta}$ from (11) and solving (11) for $\boldsymbol{\eta}$, one can formulate the control law in a more compact form as

$$
\begin{aligned}
\boldsymbol{\tau}_{m}= & \boldsymbol{\tau}_{d}+\boldsymbol{K}_{T \eta}\left(\boldsymbol{\tau}-\boldsymbol{\tau}_{d}\right)-\boldsymbol{K}_{S \eta} \boldsymbol{K}(\dot{\boldsymbol{\eta}}-\dot{\boldsymbol{q}}) \\
& +\boldsymbol{B} \boldsymbol{K}^{-1}\left(\ddot{\boldsymbol{\tau}}_{d}-\boldsymbol{D} \boldsymbol{q}^{(3)}+\boldsymbol{D} \boldsymbol{B}_{d}^{-1} \boldsymbol{D} \ddot{\boldsymbol{q}}\right) \\
\boldsymbol{K}_{T \eta}= & \boldsymbol{I}-\boldsymbol{B} \boldsymbol{B}_{d}^{-1}+\left(\boldsymbol{B} \boldsymbol{K}^{-1} \boldsymbol{D} \boldsymbol{B}_{d}^{-1}\right) \boldsymbol{D} \boldsymbol{B}_{d}^{-1} \\
\boldsymbol{K}_{S \eta}= & \boldsymbol{B} \boldsymbol{K}^{-1} \boldsymbol{D} \boldsymbol{B}_{d}^{-1}
\end{aligned}
$$

Equation (14) implements the desired dynamics (9)-(10) and leads to damping of the torque dynamics in a controllable way using the parameter $D$, as illustrated in Fig. 4 (bottom). The implementation of (14) appears to require dynamic state feedback to compute the virtual motor velocity $\dot{\eta}$. However, as we will show in the next section static feedback is sufficient.

\section{Practical realization of the torque control loop}

Accurate measurement of the torque is crucial for the performance of the low-level torque control. Obtaining it through the motor and link positions following $\boldsymbol{\tau}=\boldsymbol{K}(\boldsymbol{\theta}-\boldsymbol{q})$ can be unsatisfactory as the accuracy is highly dependent on the model $\boldsymbol{K}$ of the joint stiffness value and the resolution of the position sensors. Therefore, the control law will be reformulated in the following to take advantage of the available torque sensors in SARA as direct source of feedback. Based on (13), it is possible to eliminate the dependency on the virtual coordinate $\boldsymbol{\eta}$ by substitution. Solving (10) and (11) gives

$$
\ddot{\boldsymbol{\eta}}=\boldsymbol{B}_{d}^{-1}\left(\boldsymbol{\tau}_{d}-\boldsymbol{\tau}\right)
$$

By substituting in (13) this results in

$$
\begin{aligned}
\boldsymbol{\tau}_{m}= & \boldsymbol{\tau}_{d}+\boldsymbol{K}_{T}\left(\boldsymbol{\tau}_{d}-\boldsymbol{\tau}\right)+\boldsymbol{K}_{S}\left(\dot{\boldsymbol{\tau}}_{d}-\dot{\boldsymbol{\tau}}\right) \\
& +\boldsymbol{B} \boldsymbol{K}^{-1}\left(\ddot{\boldsymbol{\tau}}_{d}-\boldsymbol{D} \boldsymbol{q}^{(3)}\right)
\end{aligned}
$$

where $\boldsymbol{K}_{T}=\boldsymbol{B} \boldsymbol{B}_{d}^{-1}-\boldsymbol{I}$ and $\boldsymbol{K}_{S}=\boldsymbol{B} \boldsymbol{K}^{-1} \boldsymbol{D} \boldsymbol{B}_{d}^{-1}$ are the control gains. The torque derivative appears in (16) as a result of the introduced damping that acts in parallel to the spring (joint elasticity). Now any controller designed for a rigidbody robot can be applied through $\tau_{d}, \dot{\tau}_{d}, \ddot{\tau}_{d}$ in combination with (16). Notably, the proposed control structure reduces the motor-side disturbances, such as the gear-inherent friction ${ }^{7}$, with the scaling ratio of the apparent inertia.

In principle, (16) represents a torque PD tracking controller with additional terms but it is derived based on a desired dynamical behavior with physically intuitive components, as illustrated in Fig. 4. The controller structure

\footnotetext{
${ }^{6}$ The third time derivative of $\boldsymbol{\eta}$ is denoted as $\boldsymbol{\eta}^{(3)}$, the same applies for the link-side jerk $\boldsymbol{q}^{(3)}$.

${ }^{7}$ The torque feedback loop reduces the motor-side friction. The effect is scaled down by $\boldsymbol{B}_{d} \boldsymbol{B}^{-1}$ which makes robot joints with high reduction ratios backdrivable.
}

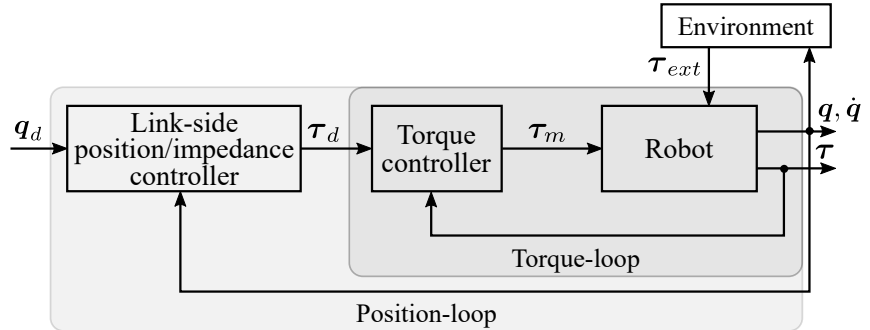

Fig. 5: The cascaded control structure for achieving link-side impedance/position control. The inner torque control loop realizes the desired torque dynamics and provides a torque interface for the outer link-side control loop.

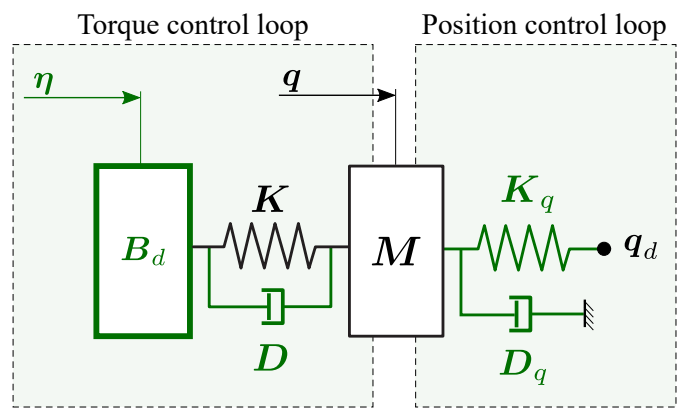

Fig. 6: Physical representation of the desired behavior of link-side impedance/position control (17) interfaced with torque joint-level controller (16).

has several similar features as the torque decoupling control approach [19] since it contains the first and the second derivative of the desired torque. However, it is derived in a different way using a direct PD control law to stabilize the torque dynamics, while here, the controller is derived in a more natural manner based on physical desired dynamics. That, in turn, makes it more intuitive to parameterize. The torque control loop can be specified through the desired damping $D$ and the ratio of the reduction of the apparent motor inertia $\boldsymbol{B} \boldsymbol{B}_{d}^{-1}$ in an intuitive way. Additional feedforward terms are needed to achieve the desired performance. The implementation of the control law does not require dynamic state feedback, that is, it does not contain additional internal states. The quantity $\boldsymbol{q}^{(3)}$ can be obtained in a model-based way, that is, as a function of $\boldsymbol{\tau}, \dot{\boldsymbol{\tau}}, \boldsymbol{q}$ and $\dot{\boldsymbol{q}}[1]^{8}$.

\section{Link-side impedance and position control}

An outer control loop can be synthesized as a link-side position or impedance control, based on the choice of the gains. In this case, the control structure can be classified as cascaded, with an inner torque control loop and an outer position/impedance loop, see Fig. 5. The graphical representation of the desired closed loop is shown in Fig. 6. This is achieved by replacing $\tau_{d}$ in (16) or (8) by the following expression:

$$
\boldsymbol{\tau}_{d}=-\boldsymbol{K}_{q}\left(\boldsymbol{q}-\boldsymbol{q}_{d}\right)-\boldsymbol{D}_{q} \dot{\boldsymbol{q}}+\boldsymbol{g}(\boldsymbol{q})
$$

\footnotetext{
${ }^{8}$ The link-side position $\boldsymbol{q}$ is measured and $\boldsymbol{q}$ is obtained via numerical differentiation of the measured position signal, a low-pass filter can be applied to limit noise effects.
} 
with $\boldsymbol{K}_{q}$ and $\boldsymbol{D}_{q}$ being the link-side stiffness and damping. That basically constitutes a joint-space regulation controller applied to the link side. With this control structure one can see that the link stiffness equals the effective stiffness $\boldsymbol{K}_{e q}=\boldsymbol{K}_{q}$. Unlike (17) the effective stiffness of the control structure (4) is a function of the joint stiffness, $\boldsymbol{K}_{e q}=\left(\boldsymbol{K}_{\theta}^{-1}+\boldsymbol{K}^{-1}\right)^{-1}$, which makes the direct interaction with the environment highly dependent on the value of the joint stiffness. Here we compare with the classical implementation of cascaded control of lightweight robots. However, there are other formulations of the control law [5] which use the estimated link-side position. In this case the performance heavily relies on the accuracy of the knowledge of the joint stiffness, which is known to be difficult to obtain in practice. The method presented here imposes no constraints on the closed-loop link-side stiffness.

\section{PASSIVITY AND STABILITy ANALYSIS}

In this section, the passivity and stability properties of the proposed control approaches are investigated.

\section{A. Link-side torque interface and motor inertia shaping}

Consider the closed-loop motor and link dynamics described by (5)-(6). A suitable storage function for the link side can be chosen as

$$
S_{q}=\frac{1}{2} \dot{\boldsymbol{q}}^{T} \boldsymbol{M}(\boldsymbol{q}) \dot{\boldsymbol{q}}+\frac{1}{2} \tilde{\boldsymbol{q}}^{T} \boldsymbol{K}_{q} \tilde{\boldsymbol{q}}
$$

where $\tilde{\boldsymbol{q}}=\boldsymbol{q}-\boldsymbol{q}_{d}$ is the link position error. Its time derivative can be straightforwardly derived as

$$
\dot{S}_{q}=\dot{\boldsymbol{q}}^{T} \boldsymbol{\tau}_{\eta}-\dot{\boldsymbol{q}}^{T} \boldsymbol{D}_{q} \dot{\boldsymbol{q}}+\dot{\boldsymbol{q}}^{T} \boldsymbol{\tau}_{e x t}
$$

Herein,

$$
\boldsymbol{\tau}_{\eta}=\boldsymbol{K}(\boldsymbol{\eta}-\boldsymbol{q})
$$

is the virtual torque transmitted through the joint in case of motor inertia shaping and introduction of the link-side torque interface. Similarly, a storage function for the motor side can be formulated as

$$
S_{\eta}=\frac{1}{2} \dot{\boldsymbol{\eta}}^{T} \boldsymbol{B}_{d} \dot{\boldsymbol{\eta}}+\frac{1}{2}(\boldsymbol{\eta}-\boldsymbol{q})^{T} \boldsymbol{K}(\boldsymbol{\eta}-\boldsymbol{q})
$$

which includes the kinetic energy related to the motor side, and the virtual elastic potential between motor and link. Its time derivative is given by

$$
\dot{S}_{\eta}=-\dot{\boldsymbol{q}}^{T} \boldsymbol{\tau}_{\eta}
$$

With the following storage function, that is,

$$
\begin{aligned}
& S=S_{q}+S_{\eta}, \\
& \dot{S}=-\dot{\boldsymbol{q}}^{T} \boldsymbol{D}_{q} \dot{\boldsymbol{q}}+\dot{\boldsymbol{q}}^{T} \boldsymbol{\tau}_{\text {ext }},
\end{aligned}
$$

one can describe the closed-loop dynamics as a passive mapping for input $\boldsymbol{\tau}_{\text {ext }}$ and output $\dot{\boldsymbol{q}}$. In the presence of motor-side friction an additional dissipative term will appear in (22).

In Fig. 7, the feedback interconnection of the two passive subsystems, related to (virtual) motor and link side, respectively, is illustrated in the shaded block. Moreover, this controlled system is feedback-interconnected to the

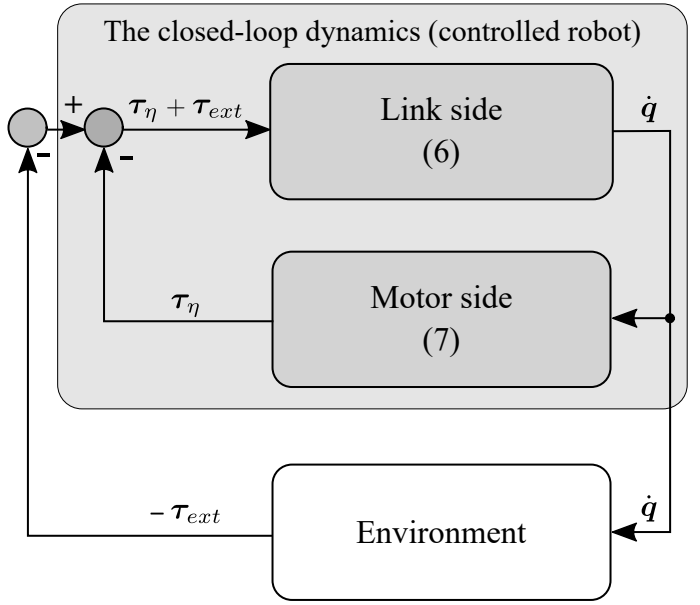

Fig. 7: Block diagram representation for the interconnection of the passive subsystems. In case of shaping the motor inertia and providing link side torque interface the virtual joint torque is $\boldsymbol{\tau}_{\eta}=\boldsymbol{K}(\boldsymbol{\eta}-\boldsymbol{q})$ and if a desired relative damping is added the virtual joint torque becomes $\tau_{\eta}=\boldsymbol{K}(\boldsymbol{\eta}-\boldsymbol{q})+\boldsymbol{D}(\dot{\boldsymbol{\eta}}-\dot{\boldsymbol{q}})$.

environment, resulting in a passive system again. Note that any passive environment can be represented as a passive mapping with $\left(\dot{\boldsymbol{q}} \rightarrow-\boldsymbol{\tau}_{\text {ext }}\right)$, see [4].

The following stability considerations address the interaction-free case, that is, $\boldsymbol{\tau}_{e x t}=\mathbf{0}$. Since $S(\boldsymbol{q}, \dot{\boldsymbol{q}}, \boldsymbol{\eta}, \dot{\boldsymbol{\eta}})$ from (23) is positive definite and radially unbounded, and its time derivative (24) is negative semi-definite when $\tau_{e x t}=\mathbf{0}$ holds, one can apply LaSalle's invariance principle to conclude global asymptotic stability of the equilibrium $(\boldsymbol{q}, \dot{\boldsymbol{q}}, \boldsymbol{\eta}, \dot{\boldsymbol{\eta}})=\left(\boldsymbol{q}_{d}, \mathbf{0}, \boldsymbol{q}_{d}, \mathbf{0}\right)$ in a straightforward way.

\section{B. Link-side torque interface, motor inertia shaping, and relative damping}

Analogous to Section IV-A, one can can proceed for the dynamics (9)-(10). The storage functions $S_{q}$ and $S_{\eta}$ can be adopted from (18) and (21), respectively. However, the virtual elastic torque transmitted through the joint is now given by

$$
\boldsymbol{\tau}_{\eta}=\boldsymbol{K}(\boldsymbol{\eta}-\boldsymbol{q})+\boldsymbol{D}(\dot{\boldsymbol{\eta}}-\dot{\boldsymbol{q}})
$$

due to the additional relative damping that is actively injected. When choosing (23) as the total storage function, one can determine its time derivative as

$$
\dot{S}=-(\dot{\boldsymbol{\eta}}-\dot{\boldsymbol{q}})^{T} \boldsymbol{D}(\dot{\boldsymbol{\eta}}-\dot{\boldsymbol{q}})-\dot{\boldsymbol{q}}^{T} \boldsymbol{D}_{q} \dot{\boldsymbol{q}}+\dot{\boldsymbol{q}}^{T} \boldsymbol{\tau}_{e x t}
$$

Intuitively speaking, the closed-loop system for Section IVB is also passive with respect to the storage function $S$, the input $\tau_{\text {ext }}$, and the output $\dot{\boldsymbol{q}}$, but with an additional damping element compared to Section IV-A. This additional source of energy dissipation is expected to be beneficial in terms of vibration damping because oscillations on the (virtual) motor side can be directly damped out.

Analogous to the stability considerations in Section IV-A, one can proceed for the case in Section IV-B. By means of LaSalle's invariance principle, one can show that the equilibrium $(\boldsymbol{q}, \dot{\boldsymbol{q}}, \boldsymbol{\eta}, \dot{\boldsymbol{\eta}})=\left(\boldsymbol{q}_{d}, \mathbf{0}, \boldsymbol{q}_{d}, \mathbf{0}\right)$ is globally asymptotically stable for the interaction-free case $\left(\boldsymbol{\tau}_{\text {ext }}=\mathbf{0}\right)$. 


\section{EXPERIMENTS}

The proposed control method can be classified as a cascaded structure. However, in the real robot implementation, both control loops are running at the same sampling rate. The low-level joint torque control loop and the impedance/position controller are running at $8 \mathrm{kHz}$ in the DLR SARA robot. The high sampling rate allows us to achieve a link-side impedance/position control gain as high as the intrinsic joint stiffness. The experimental validation is conducted using the fifth joint of the DLR SARA system with a maximum torque of $57 \mathrm{Nm}$. The controller with relative damping and link-side torque interface was used in the experiments as it is more robust from the realization point of view, and it allows to damp-out local joint vibration. The torque control gains are calculated based on the shaping ratio of the apparent motor inertia $\boldsymbol{i}=\boldsymbol{B} \boldsymbol{B}_{d}^{-1}$ and the damping parameterization $\boldsymbol{D}$ throughout all the experiments. The parameters of the considered joint are $b_{5}=0.339 \mathrm{kgm}^{2}$ (motor inertia), $k_{5}=9000 \mathrm{Nm} / \mathrm{rad}$ (intrinsic joint stiffness), and in the considered robot configuration the diagonal element of the inertia matrix is $m_{5,5}=0.56 \mathrm{kgm}^{2}$.

\section{A. SARA system description}

The KUKA-DLR LWR-III lightweight robots are established in various applications of research and industry by now [2]. With the experience from that, the DLR has developed a new generation of DLR lightweight robots that bear the name SARA (Safe Autonomous Robotic Assistant). It is expected to push the technological limits far beyond the state of the art and enable new fields of application. In SARA, the motor and link position measurements are available throughout highresolution encoders of a magnetoresistive type that represent the position before and after the joint deflection. Moreover, the joint torque measurement is provided throughout straingauge-based torque sensors with optimized design to increase the sensor resolution and stiffness, and this represents the elastic torque across the joint flexibility. Using these sensors, different combinations of state feedback controllers can be realized. Since $\tau$ is directly measured through a highresolution (16-bit) joint torque sensor, $\dot{\tau}$ can be obtained smoothly using numerical differentiation. ${ }^{9}$

\section{B. Static and dynamic deflections: load-dependent position error}

The performance of the high gain position/impedance controller is validated using a trajectory that contains free movements and standstill phases in order to show the static and dynamic deflections during free motion. Fig. 8 shows the experimental results of the two different controllers (3)(4) and (16)-(17) while moving along a smooth trajectory with an amplitude of $\pm 90^{\circ}$. During this motion a load of $3.85 \mathrm{~kg}$ was attached to the end-effector. This represents about one-third of the payload of the robot. The deviation corresponds to two effects due to joint flexibility. The first corresponds to static deflection, which can be seen in the standstill phases of the trajectory. The second is the dynamic

\footnotetext{
${ }^{9} \mathrm{~A}$ first-order low-pass filter is applied to the obtained signal of $\dot{\tau}$ with cutoff frequency $(500 \mathrm{~Hz})$ for SARA robot.
}
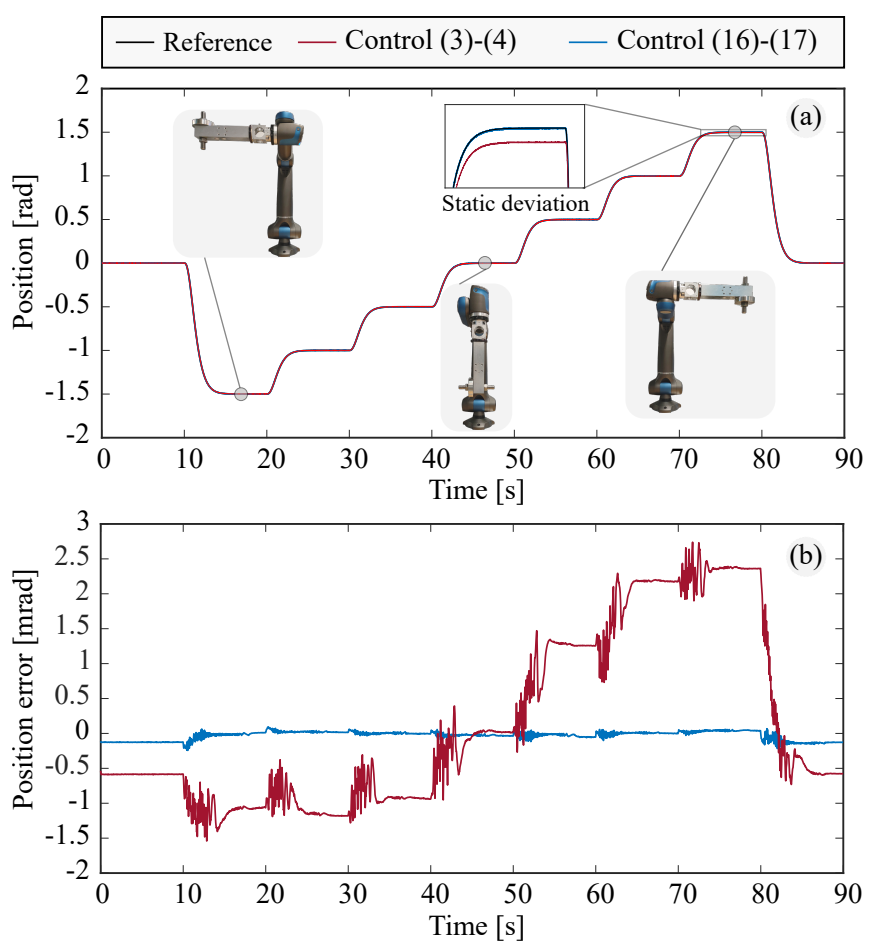

Fig. 8: The experimental result of an executed trajectory from $-90^{\circ}$ to $+90^{\circ}$ in smooth steps is shown in (a). The dynamic deflections during motion phases are shown in (b) and also the static deflections during the standstill phases. The controller (3)-(4) refers to the typical implementation in the DLR-LWR robots [20].

deflection that appears during motion. This can be seen as the oscillatory behavior in Fig. 8. Figure 8.b illustrates the considerable improvement of the transient behavior as well as the steady-state error in terms of the link-side position error. To have a fair performance comparison, the gravity compensation action is computed based on the motor position with both controllers, as in this range of position differences, gravitational effects will not affect the results in a noticeable way. In other words, the difference in the gravitational torque will be less than the modeling uncertainty. Due to the harmonic drive the joint stiffness behavior is weakly nonlinear and asymmetric. Figure 9 provides a closer look at the link-side vibration damping during the motion phase and the compensation of static deflection compared to the classical motor-side control.

\section{Vibration damping / stiff position control}

One experiment is carried out to validate the link-side damping capabilities. As shown in Fig. 10 a combined trajectory that contains $0.01 \mathrm{rad}$ steps is commanded with a limited rate in order not to violate the continuity property of the desired torque. The step response of the two control structures (3),(4) and (16),(17) employed as a stiff (highgain) position controller is illustrated in Fig. 10.b. The position control gains are the same in the two cases and the control gains of the torque control loop are computed based on the same damping value $D$ and the reduction ratio of the apparent motor inertia. The highlighted peaks in Fig. 10.b. show an improvement of approximately 50\% 


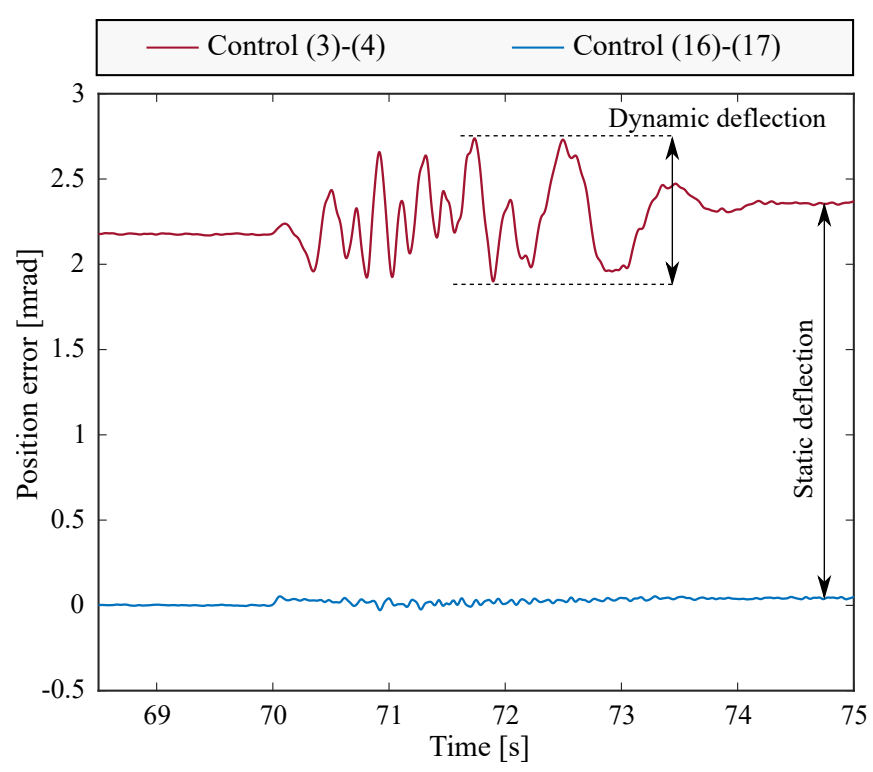

Fig. 9: Zoomed view of a part from Fig. 8. The effect of the dynamic deflection (during motion phase) and the static deflection (at standstill phase) due to the joint elasticity in terms of linkside position error. The link-side position control corresponds to (16),(17) and the motor-side position control corresponds to (3),(4).

for the proposed link-side controller compared to the state of the art [5]. The fast sampling rate in SARA allows high controller gains comparable to the value of the intrinsic joint stiffness. As a result, the stiff link-side controller can achieve near-zero link-side position error, see Fig. 10. The ability to use the same control structure with low and high gains is more convenient to apply position (stiff) or compliant control, by changing the control gains without the need to switch between different controllers. In other words, a desired impedance behavior can be implemented, and continuously increasing the corresponding stiffness gain
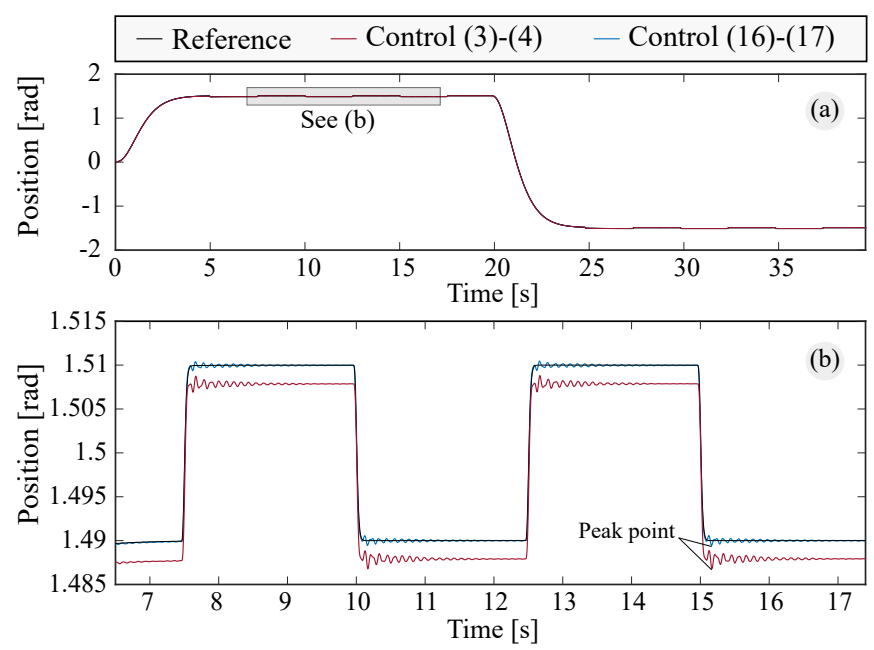

Fig. 10: Step response of the link side with (16)-(17) and motor side with (3)-(4) torque/position controllers. In (a) the trajectories that contains $0.01 \mathrm{rad}$ steps is illustrated. A closer look at the step response and the oscillatory behavior of each signal is shown in (b).
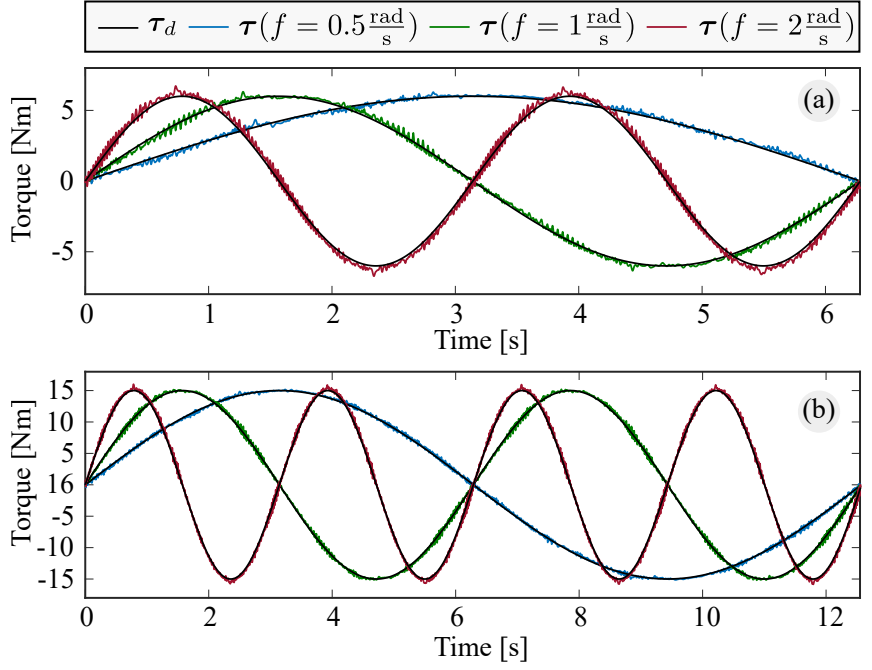

Fig. 11: The tracking performance of the torque control loop in case of applying the controller with relative damping term (16). The desired and measured torques are shown with three different frequencies. The desired torque amplitude is set to $6 \mathrm{Nm}$ in (a) and $15 \mathrm{Nm}$ in (b).

will ultimately result in a stiff position control, while the joint-level vibration-damping is kept active all the time.

\section{Torque tracking (open outer-loop)}

The experimental validation of the torque control loop is conducted by commanding a sinusoidal desired torque to evaluate the tracking performance. Figure 11 depicts the response of the torque signal in case of using the control law (16) with three different frequencies and two amplitudes. Similarly, the control law (3) is tested applying the same signals, the proposed approach (16) shows a reduced of the root mean square error (RMSE) by $\approx 7-10 \%$ over the classical torque controller. The torque tracking performance of both controllers is close. This not surprising as both controllers have damping terms for the torque dynamics. More intuitively, in (16) the control law is derived based on the desired dynamic behavior that generalizes the method to extract the control law by assuming any other behavior for the torque control loop. Moreover, the torque tracking performance with relatively low amplitude that is depicted in Fig. 11 (top) implies the ability to achieve a link-side compliant behavior (low impedance).

\section{CONCLUSIONS AND FUtURE WORKS}

A method to derive a joint-torque controller for lightweight robots based on a desired dynamical behavior was presented. Two cases were investigated. The first one aimed at providing a link-side torque interface and actively shaping the motor inertia. The second one also achieved these two goals but additionally featured active damping between motor and link coordinates to suppress vibrations. The final control formulation intuitively reflected the physics which is advantageous in terms of easy controller parameterization. The implementation of the control law does not require dynamic state feedback as it only depends on measurable states. The proposed method was tested on the DLR SARA robot 
with high sampling rate which makes it possible to achieve such a high link-side stiffness in practice. Experiments with an outer-loop, stiff position controller were shown and the torque tracking performance was experimentally evaluated. Significant improvements in the static and dynamic link-side position errors were also achieved compared to the state of the art.

In the next step the link-side torque interface will be utilized to realize a coordinated, impedance-based multipriority control behavior [21]-[23] with focus on highly dynamic task trajectory tracking [24].

\section{REFERENCES}

[1] A. De Luca and W. J. Book, "Robots with flexible elements," in Springer Handbook of Robotics. Springer, 2016, pp. 243-282.

[2] G. Hirzinger, N. Sporer, A. Albu-Schäffer, M. Hahnle, R. Krenn, A. Pascucci, and M. Schedl, "DLR's torque-controlled light weight robot III-are we reaching the technological limits now?" in Proc. of the 2002 IEEE International Conference on Robotics and Automation, May 2002, pp. 1710-1716.

[3] R. EMIKA., "This is Franka, [online]. available: https://www.franka.de/,” Jan. 23, 2017.

[4] N. Hogan, "Impedance Control: An Approach to Manipulation: Part I - Theory, Part II - Implementation, Part III - Applications," Journal of Dynamic Systems, Measurement, and Control, vol. 107, pp. 1-24, March 1985

[5] A. Albu-Schäffer, C. Ott, and G. Hirzinger, "A unified passivitybased control framework for position, torque and impedance control of flexible joint robots," The international journal of robotics research, vol. 26, no. 1, pp. 23-39, 2007.

[6] P. Tomei, "A simple pd controller for robots with elastic joints," Automatic Control, IEEE Transactions on, vol. 36, no. 10, pp. 12081213, Oct 1991.

[7] R. Ortega, R. Kelly, and A. Loria, "A class of output feedback globally stabilizing controllers for flexible joints robots," Robotics and Automation, IEEE Transactions on, vol. 11, no. 5, pp. 766-770, Oct 1995.

[8] L. Zollo, A. De Luca, and B. Siciliano, "Regulation with on-line gravity compensation for robots with elastic joints," in Robotics and Automation, 2004. Proc. 2004 IEEE International Conference on Robotics and Automation, April 2004, pp. 2687-2692.

[9] C. Ott, A. Albu-Schäffer, A. Kugi, and G. Hirzinger, "On the PassivityBased Impedance Control of Flexible Joint Robots," IEEE Transactions on Robotics, vol. 24, no. 2, pp. 416-429, April 2008.

[10] I. Sardellitti, G. Medrano-Cerda, N. Tsagarakis, A. Jafari, and D. Caldwell, "Gain scheduling control for a class of variable stiffness actuators based on lever mechanisms," Robotics, IEEE Transactions on, vol. 29, no. 3, pp. 791-798, June 2013.
[11] F. Petit and A. Albu-Schäffer, "State feedback damping control for a multi dof variable stiffness robot arm," in Proc. IEEE Int. Conf. on Robotic and Automation, 2011.

[12] M. Keppler, D. Lakatos, C. Ott, and A. Albu-Schäffer, "Elastic Structure Preserving (ESP) Control for Compliantly Actuated Robots," IEEE Transactions on Robotics, vol. 34, no. 2, pp. 317-335, March 2018.

[13] — "Elastic structure preserving (espi) control of compliantly actuated robots," in 2018 IEEE International Conference on Intelligent Robots and Systems (IROS). IEEE, October 2018, pp. 5861-5868.

[14] M. W. Spong, "Modeling and Control of Elastic Joint Robots," Journal of Dynamic Systems, Measurement, and Control, vol. 109, no. 4, pp. 310-319, December 1987.

[15] S. Wolf and M. Iskandar, "Extending a dynamic friction model with nonlinear viscous and thermal dependency for a motor and harmonic drive gear," in 2018 IEEE International Conference on Robotics and Automation (ICRA). IEEE, May 2018, pp. 783-790.

[16] M. Iskandar and S. Wolf, "Dynamic friction model with thermal and load dependency: modeling, compensation, and external force estimation," in 2019 IEEE International Conference on Robotics and Automation (ICRA). IEEE, May 2019, pp. 7367-7373.

[17] S. Wolf and M. Iskandar, "Verfahren zur parametrischen Modellierung von Reibung und Reibungskompensation mit nichtlinearen Anhängigkeiten von Temperatur, Last und Geschwindigkeit," German patent No. DE 102019112 611, patented on May 15, 2019.

[18] L. Le Tien, A. Albu-Schäffer, A. De Luca, and G. Hirzinger, "Friction observer and compensation for control of robots with joint torque measurement," in 2008 IEEE/RSJ International Conference on Intelligent Robots and Systems. IEEE, 2008, pp. 3789-3795.

[19] C. Ott, A. Albu-Schäffer, A. Kugi, and G. Hirzinger, "Decoupling Based Cartesian Impedance Control of Flexible Joint Robots," in Proc. of the 2003 IEEE International Conference on Robotics and Automation, September 2003, pp. 3101-3107.

[20] A. Albu-Schäffer, C. Ott, and G. Hirzinger, "A Passivity Based Cartesian Impedance Controller for Flexible Joint Robots - Part II: Full State Feedback, Impedance Design and Experiments," in Proc. of the 2004 IEEE International Conference on Robotics and Automation, April 2004, pp. 2666-2672.

[21] A. Dietrich, T. Wimböck, A. Albu-Schäffer, and G. Hirzinger, "Reactive Whole-Body Control: Dynamic Mobile Manipulation Using a Large Number of Actuated Degrees of Freedom," IEEE Robotics \& Automation Magazine, vol. 19, no. 2, pp. 20-33, June 2012.

[22] A. Dietrich, C. Ott, and A. Albu-Schäffer, "An overview of null space projections for redundant, torque-controlled robots," International Journal of Robotics Research, vol. 34, no. 11, pp. 1385-1400, Sept 2015.

[23] M. Iskandar, G. Quere, A. Hagengruber, A. Dietrich, and J. Vogel, "Employing whole-body control in assistive robotics," in IEEE International Conference on Intelligent Robots and Systems, November 2019 , pp. $5643-5650$.

[24] A. Dietrich and C. Ott, "Hierarchical Impedance-Based Tracking Control of Kinematically Redundant Robots," IEEE Transactions on Robotics, vol. 36, no. 1, pp. 204-221, February 2020. 